\title{
Seniority-conserving forces and $\mathrm{USp}(2 j+1)$ partial dynamical symmetry
}

\author{
G. Rosensteel \\ Department of Physics, Tulane University, New Orleans, Louisiana 70118 \\ D. J. Rowe \\ Department of Physics, University of Toronto, Toronto, Ontario, Canada M5S 1A7
}

(Received 14 October 2002; published 16 January 2003)

\begin{abstract}
A quasispin tensor decomposition of the two-nucleon interaction determines the most general seniorityconserving rotationally invariant two-body interaction in a $j$ shell. Such interactions define solvable and partially solvable shell model Hamiltonians for which the unitary symplectic algebra USp $(2 j+1)$ provides a complete set of quantum numbers for a subset of states. The matrix elements of seniority-conserving interactions are deduced from the matrix elements of $\operatorname{USp}(2 j+1)$ operators. A new and powerful numerical technique is presented for computing irreps of the $\operatorname{USp}(2 j+1)$ algebra. Applications are reported for the lowenergy spectra of $N=50$ and $N=126$ isotones. The effects of including seniority nonconserving interactions are investigated.
\end{abstract}

DOI: 10.1103/PhysRevC.67.014303

PACS number(s): 21.60.Fw

\section{INTRODUCTION}

The objective of this paper is to explore the remarkable successes and simplifications that can be achieved in a model description of singly-closed shell nuclei with seniority conserving interactions restricted to a single $j$ shell. For such interactions, many low-energy states are uniquely defined by seniority and angular momentum quantum numbers. Thus the model has a partial dynamical symmetry according to the definition of Alhassid and Leviatan [1]. The seniority coupling scheme and the properties of seniority-conserving Hamiltonians are given in Talmi's book [2]. In Sec. II we provide a brief summary of those parts of the theory needed for present purposes. Our approach makes extensive use of the duality relationship, observed by Helmers [3], between the classification of states by seniority and by quasispin. A preliminary account of part of the current study was presented in Ref. [4].

When identical fermions occupy a single $j$ shell, monopole pairing dominates the nucleon-nucleon interaction. The energy spectrum of a closed-shell-plus-two-proton (or twoneutron) nucleus, such as ${ }^{92} \mathrm{Mo}$ or ${ }^{210} \mathrm{Po}$, in which the energy of the $J=0$ ground state is substantially below the energies of the $J \neq 0$ excited states, shows that monopole pairing is a major influence.

The quasispin algebra $\mathrm{SU}(2)_{S}$ is a spectrum generating algebra for the monopole pairing interaction in the sense that this interaction is a polynomial in the elements of $\mathrm{SU}(2)_{S}$. In this case, the irreducible representations (irreps) of $\mathrm{SU}(2)_{S}$ determine analytically the spectrum of the Hamiltonian. The $\mathrm{SU}(2)_{S}$ irreps are labeled by a total quasispin quantum number $S=(\Omega-v) / 2$, where $\Omega=(2 j+1) / 2$ and $v$ denotes the seniority (the number of unpaired nucleons) [2]. Excitation energies of the monopole pairing quasispin model depend only on the total quasispin or equivalently the seniority. However, while the model explains some gross features of many singly-closed shell nuclei, it provides a poor description of the details.

The quasispin model can be improved by introducing in- teractions that split the degeneracies of same-seniority states but do not mix states of different seniority. Let $|n v \rho J M\rangle$ denote a basis for the space of $n$ identical fermions in a $j$ shell, where $v$ is the seniority and $\rho$ is the multiplicity of the angular momentum $J M$. When the Hamiltonian $\hat{H}$ is rotationally invariant and seniority conserving, the angular momentum and seniority are good quantum numbers:

$$
\left\langle n v^{\prime} \rho^{\prime} J^{\prime} M^{\prime}|\hat{H}| n v \rho J M\right\rangle=\delta_{v^{\prime} v} \delta_{J^{\prime} J} \delta_{M^{\prime} M} E_{\rho^{\prime} \rho}(n v J) .
$$

In the simple quasispin model the matrix $E_{\rho^{\prime} \rho}(n v J)$ is diagonal and independent of $J$.

As shown by Talmi [2], any seniority-conserving twobody interaction is the sum of a monopole pairing interaction and a quadratic operator in the compact unitary symplectic algebra, $\operatorname{USp}(2 j+1)$. The operators of $\operatorname{USp}(2 j+1)$ commute with the quasispin generators. In fact, $\operatorname{USp}(2 j+1)$ is the maximal subalgebra of $\mathrm{U}(2 j+1)$ having this property, where $\mathrm{U}(2 j+1)$ is the algebra of all hermitian one-body operators that act in a single $j$ shell. As a result, $\operatorname{USp}(2 j$ $+1)$ serves to classify all the states of a single $j$-shell nucleus of a given seniority [5,3]; indeed seniority is a label for a $\operatorname{Usp}(2 j+1)$ irrep. Moreover, the representation theory of $\operatorname{USp}(2 j+1)$ provides an algebraic framework for diagonalizing any seniority conserving Hamiltonian.

The intimate relationship between the commuting $\mathrm{USp}(2 j+1)$ and $\mathrm{SU}(2)_{S}$ algebras [3] is an example of a duality relationship $[6,7]$. A familiar example of such a relationship (also known as complementarity in the physics literature [8]) is the famous Schur-Weyl duality of the permutation group $S_{p}$ and the unitary group $\mathrm{U}(\mathrm{m})$ when acting in the tensor product space of $p$ copies of an $m$-dimensional vector space. A consequence of this duality is that the representations of the permutation and unitary groups intertwine and both are labeled uniquely by Young diagrams.

Duality within a $j$-shell implies similarly that the irreps of the quasispin algebra and the irreps of the unitary symplectic algebra are in one-to-one correspondence. The common label 
TABLE I. Irreducible representations of $\mathrm{SU}(2)_{S} \times \mathrm{USp}(2 j+1)$ for $j=7 / 2$.

\begin{tabular}{lrrrrrr}
\hline \hline Irrep & $n$ & \multicolumn{1}{c}{$S_{0}$} & \multicolumn{5}{c}{$J$} \\
\hline$v=4$ & 4 & 0 & 2 & 4 & 5 & 8 \\
$S=0$ & & & & & & \\
$v=2$ & 2 & -1 & 2 & 4 & 6 & \\
$S=1$ & 4 & 0 & 2 & 4 & 6 & \\
& 6 & 1 & 2 & 4 & 6 & \\
& & & & & & \\
$v=0$ & 0 & -2 & 0 & & & \\
$S=2$ & 2 & -1 & 0 & & & \\
& 4 & 0 & 0 & & & \\
& 6 & 1 & 0 & & & \\
& 8 & 2 & 0 & & & \\
& & & & & & \\
\hline \hline
\end{tabular}

for the $\operatorname{SU}(2)_{S}$ and $\mathrm{USp}(2 j+1)$ irreps is the seniority $v$. As an example, Table I organizes the basis states of even seniority in the $j=\frac{7}{2}$ shell with respect to $\mathrm{SU}(2)_{S}$ and $\mathrm{USp}(2 j$ $+1)$. Each seniority $v$ in the table labels a unitary irrep of the compact symplectic algebra USp(8). A basis of good angular momentum states that spans this representation is indicated by reading the table horizontally, e.g., for $v=4$, the $\mathrm{USp}(8)$ irrep contains states of $J=2,4,5,8$. For $v=2$, there are three equivalent $\mathrm{USp}(8)$ irreps, each with $J=2,4,6$. For $v=0$, there are five equivalent identity $(J=0) \operatorname{USp}(8)$ irreps. In addition, each seniority $v$ in the table determines a unique dual irrep of the quasispin algebra $\mathrm{SU}(2)_{S}$ labeled by the quasispin $S$. The angular momentum is constant within a quasispin irrep, and the irrep's basis is found by reading vertically the table entries. Thus, there are five $v=0, J=0$ states which span a quasispin $S=2$ irrep. For $v=2$, there are three equivalent sets of three-dimensional $S=1$ irreps with $J=2,4,6$ and $M=-J, \ldots,+J$, and, when $v=4$, there are four equivalent sets of one-dimensional $S=0$ irreps. The " $\hat{S}_{0}$ " component of the quasispin algebra is related linearly to the number operator by $\hat{S}_{0}=(\hat{n}-\Omega) / 2$.

Within a single $j$ shell, there are $\Omega$ linearly independent two-body rotationally invariant interactions. However, the number of interactions that mix seniority is much smaller for the values of $j$ that occur in nuclear physics. In fact, it is known from the work of Racah in atomic spectroscopy [9] that when $j \leqslant \frac{7}{2}$ every two-body rotational-scalar interaction conserves seniority. This is clear from Table I which shows that states of a given $n v$ and $J M$ are multiplicity free for a $j=7 / 2$ shell configuration. The conditions that the matrix elements of a general interaction must satisfy to conserve seniority have been given by Talmi [2]. These conditions were rediscovered in Ref. [4] and used to construct linearlyindependent combinations of the $\Omega$ interactions such that all but one conserve seniority for $\frac{9}{2} \leqslant j \leqslant \frac{13}{2}$; all but two conserve seniority for $\frac{15}{2} \leqslant j \leqslant \frac{19}{2}$. The explicit number of quasispin scalar interactions and the number of seniority-mixing interactions for any $j$ shell is given in Sec. III in confirmation of a result inferred previously by Talmi [2] from a criterion of de-Shalit and Talmi [10] and a number-of-states identity derived by Ginocchio and Haxton [11].

Calculating the matrix elements of rotationally invariant two-body operators in the $\operatorname{USp}(2 j+1)$ algebra is a nontrivial task because the angular momentum algebra $\mathrm{SU}(2)_{J}$ is not embedded canonically in $\operatorname{USp}(2 j+1)$. Fractional parentage coefficients, when they are known, provide a tool for calculating such states and matrix elements. However, to calculate fractional parentage coefficients is to compute the matrix elements of an even larger fermion-pair algebra. As shown in Sec. IV, a recursive method of defining states of good angular momentum by stepping down from a highest weight state provides analytical results for a few high angular momentum states. But this method is also too complicated for more than a few steps and for large values of $j$. We present a simpler and more direct method for matrix element computation that can be used for low-dimensional irreps of any Lie algebra. Our technique is particularly useful when the rank of the algebra makes standard methods intractable.

The method is based on the observation that the $\operatorname{USp}(2 j$ +1 ) commutation relations define an overdetermined system of nonlinear equations for the unknown matrix elements. The fundamental theorem of highest weight representations guarantees that a solution to this system exists. In spite of the fact that the number of equations and the number of unknowns are large, we are able to solve the equations for all matrix elements of a USp $(2 j+1)$ operator basis up to and including the $j=\frac{11}{2}$ shell on a small notebook computer. These matrix elements are then used to compute the eigenvalues of the most general seniority-conserving interaction.

In Sec. $\mathrm{V}$, the theory of seniority-conserving interactions is applied to the isotones ${ }^{92} \mathrm{Mo},{ }^{94} \mathrm{Ru},{ }^{96} \mathrm{Pd}$, and ${ }^{210} \mathrm{Po}$, ${ }^{212} \mathrm{Rn},{ }^{214} \mathrm{Ra}$ regarded as singly-closed proton shell nuclei with their extra-core neutrons in a $j=9 / 2$ shell. The predicted energies of excited states are an order of magnitude better than those of the monopole pairing model. In particular, the $v=4$ states of angular momentum $J=J_{\max }$ and $J=J_{\max }-2$ are observed experimentally and their measured energies are described rather well with a seniority-conserving Hamiltonian. The states of angular momentum $J_{\max }$ and $J_{\max }-2$ are always multiplicity-free in a $\operatorname{USp}(2 j+1)$ irrep and analytic formulas are reported for their energies.

A model may be solvable, for example, because its eigenstates are identified completely by the quantum numbers of a subgroup chain. Likewise a model may be partially solvable because a set of quantum numbers defines uniquely a subset of multiplicity-free states [12]. Models described by Levia$\tan [1]$ as having a partial dynamical symmetry are of the second type. Such a partial dynamical symmetry is anticipated in the seniority-conserving models when there is no angular momentum multiplicity so that the energy matrix $E(n v J)=E_{\rho^{\prime} \rho}(n v J)$ of Eq. (1) is one dimensional. However, the energies of multiplicity-free states have not been calculated analytically until now. We find analytical expressions for the energies of some multiplicity-free states in the seniority-conserving model.

The effect of seniority-mixing interactions is investigated in Sec. VI. When the seniority conservation assumption is relaxed, the interaction becomes quadratic in the $\mathrm{U}(2 j+1)$ algebra of all hermitian one-body operators in a $j$ shell 
[rather than its subalgebra $\operatorname{USp}(2 j+1)]$. The most general seniority-mixing interaction is determined using the theory of Sec. III and its matrix elements are derived using the numerical technique of Sec. IV B extended to $\mathrm{U}(2 j+1)$. The effect of seniority-mixing on the energy spectra of the isotones studied in Sec. V is found to be small, thereby confirming that seniority-conservation is a good approximation for these nuclei.

\section{FERMION LIE ALGEBRAS}

This section gives definitions of the fermion Lie algebras relevant to a $j$ shell and the duality relationships between their irreducible unitary representations. It also provides highest weight data and branching rules for these representations.

\section{A. Basic definitions}

For a single $j$ shell, let $a_{j m}^{\dagger}$ and its Hermitian adjoint $a^{j m}$ denote the creation and annihilation operators for a single nucleon (e.g., neutron) that satisfy the fermion anticommutation relations

$$
\left\{a^{j m}, a_{j n}^{\dagger}\right\}=\delta_{m n}
$$

The one-body operators

$$
\hat{C}_{n}^{m}=a_{j n}^{\dagger} a^{j m}, \quad-j \leqslant m, n \leqslant j
$$

span a Lie algebra $\mathrm{U}(2 j+1)$ of the unitary group. The angular momentum $\hat{J}_{M}$ is a one-body vector operator,

$$
\hat{J}_{M}=\sum_{m, n}\left\langle j n\left|\hat{J}_{M}\right| j m\right\rangle \hat{C}_{n}^{m}
$$

The creation and annihilation operators for time-reversed orbitals are assumed to be given by

$$
a_{j \bar{m}}^{\dagger}=(-1)^{j,-m} a_{j,-m}^{\dagger}, \quad a^{j \bar{m}}=(-1)^{j-m} a^{j,-m} .
$$

The creation operator $a_{j m}^{\dagger}$ and the destruction operator $a_{j m}$ $\equiv a^{j \bar{m}}$ are the $m$ components of spherical tensor operators (note the subscripts to indicate tensors).

Given any two spherical tensor operators, $\hat{X}_{j_{1}}$ and $\hat{Y}_{j_{2}}$, define the coupled tensor operator $\hat{W}_{J M}=\left(\hat{Y}_{j_{2}} \otimes \hat{X}_{j_{1}}\right)_{J M}$ by

$$
\hat{W}_{J M}=\sum_{m_{1}, m_{2}}\left(j_{1} m_{1}, j_{2} m_{2} \mid J M\right) \hat{Y}_{j_{2} m_{2}} \hat{X}_{j_{1} m_{1}}
$$

This right-to-left coupling convention yields the WignerEckart theorem with the usual sequence of $M$ values, i.e.,

$$
\left\langle J_{3} M_{3}\left|\hat{W}_{J_{2} M_{2}}\right| J_{1} M_{1}\right\rangle=\left(J_{1} M_{1}, J_{2} M_{2} \mid J_{M} M_{3}\right) \frac{\left\langle J_{3}\left\|\hat{W}_{J_{2}}\right\| J_{1}\right\rangle}{\left[J_{3}\right]},
$$

and the intermediate state sum formula

$$
\begin{aligned}
\left\langle J_{3}\left\|\hat{W}_{J_{2}}\right\| J_{1}\right\rangle= & {\left[J_{2}\right] \sum_{\alpha J^{\prime}} W\left(J_{1} j_{1} J_{3} j_{2} ; J^{\prime} J_{2}\right)\left\langle J_{3}\left\|\hat{Y}_{j_{2}}\right\| \alpha J^{\prime}\right\rangle } \\
& \times\left\langle\alpha J^{\prime}\left\|\hat{X}_{j_{1}}\right\| J_{1}\right\rangle,
\end{aligned}
$$

where $[J]=\sqrt{2 J+1}$ and $W(\cdots)$ is a Racah coefficient. A coupled commutator of two spherical tensors is then defined by

$$
\left[\hat{X}_{j_{1}}, \hat{Y}_{j_{2}}\right]_{J M}=\left(\hat{X}_{j_{1}} \otimes \hat{Y}_{j_{2}}\right)_{J M}-(-1)^{j_{1}+j_{2}-J}\left(\hat{Y}_{j_{2}} \otimes \hat{X}_{j_{1}}\right)_{J M}
$$

\section{B. The fermion pair algebra and its subalgebras}

The fermion pair algebra for a single $j$ shell is spanned by the pair creation and annihilation operators

$$
\begin{aligned}
& \hat{A}_{L M}=\frac{1}{\sqrt{2}}\left(a_{j}^{\dagger} \otimes a_{j}^{\dagger}\right)_{L M}, \\
& \hat{B}_{L M}=\frac{1}{\sqrt{2}}\left(a_{j} \otimes a_{j}\right)_{L M},
\end{aligned}
$$

for even $L \leqslant 2 j-1$, and the number conserving operators

$$
\begin{aligned}
\hat{C}_{L M} & =\frac{1}{2}\left[\left(a_{j}^{\dagger} \otimes a_{j}\right)_{L M}+\left(a_{j} \otimes a_{j}^{\dagger}\right)_{L M}\right], \\
& =\left(a_{j}^{\dagger} \otimes a_{j}\right)_{L M}-\sqrt{\frac{\Omega}{2}} \delta_{L, 0},
\end{aligned}
$$

for all $L \leqslant 2 j$. These operators obey the commutation relations

$$
\begin{gathered}
{\left[\hat{B}_{L_{1}}, \hat{A}_{L_{2}}\right]_{L}=2(-1)^{L}\left[L_{1}\right]\left[L_{2}\right] W\left(L_{1} j L_{2} j ; j L\right) \hat{C}_{L},} \\
{\left[\hat{C}_{L_{1}}, \hat{A}_{L_{2}}\right]_{L}=2\left[L_{1}\right]\left[L_{2}\right] W\left(L_{1} j L_{2} j ; j L\right) \hat{A}_{L},} \\
{\left[\hat{B}_{L_{1}}, \hat{C}_{L_{2}}\right]_{L}=2\left[L_{1}\right]\left[L_{2}\right] W\left(L_{1} j L_{2} j ; j L\right) \hat{B}_{L},} \\
{\left[\hat{C}_{L_{1}}, \hat{C}_{L_{2}}\right]_{L}=\left[(-1)^{L_{1}+L_{2}-L}-1\right]\left[L_{1}\right]} \\
\times\left[L_{2}\right] W\left(L_{1} j L_{2} j ; j L\right) \hat{C}_{L},
\end{gathered}
$$

where $[L] \equiv \sqrt{2 L+1}$. This algebra is the Lie algebra $\mathrm{O}(4 j$ $+2)$ of a group of orthogonal transformations of a $(4 j+2)$-dimensional vector space of operators spanned by the fermion creation and annihilation operators.

The fermion pair algebra has several subalgebras of use for classifying states of a single $j$ shell. In particular, the subset of all number conserving operators $\left\{\hat{C}_{L M}\right\}$ span a $\mathrm{U}(2 j+1)$ algebra and the subset of $L=0$ operators $\left\{\hat{A}_{0}, \hat{B}_{0}, \hat{C}_{0}\right\}$ span the so-called quasispin algebra $\mathrm{SU}(2)_{S}$. Equivalently, $\mathrm{SU}(2)_{S}$ is spanned by the quasispin operators 


$$
\begin{gathered}
\hat{S}_{+}=\sum_{m=1 / 2}^{j} a_{j m}^{\dagger} a_{j m}^{\dagger}, \quad \hat{S}_{-}=\sum_{m=1 / 2}^{j} a^{j \bar{m}} a^{j m}, \\
\hat{S}_{0}=\frac{1}{2}(\hat{n}-\Omega)=\sqrt{\frac{\Omega}{2}} \hat{C}_{0},
\end{gathered}
$$

which satisfy the usual $\mathrm{SU}(2)$ commutation relations

$$
\left[\hat{S}_{+}, \hat{S}_{-}\right]=2 \hat{S}_{0}, \quad\left[\hat{S}_{0}, \hat{S}_{ \pm}\right]= \pm \hat{S}_{ \pm} .
$$

The $\mathrm{U}(2 j+1)$ subalgebra is useful because all the states of a given nucleon number belong to a single $\mathrm{U}(2 j+1)$ irrep. The quasispin algebra is useful because it is a spectrum generating algebra for the standard monopole pairing interaction

$$
G \sum_{m n} a_{j m}^{\dagger} a_{j m}^{\dagger} a^{j \bar{n}} a^{j n}=G \hat{S}_{+} \hat{S}_{-} .
$$

\section{Dual pairs of subalgebras of $\mathrm{O}(4 j+2)$}

As subalgebras of $\mathrm{O}(4 j+2)$, the elements of $\mathrm{U}(2 j+1)$, and $\mathrm{SU}(2)_{S}$ do not commute with one another. Thus, it is useful to consider mutually commuting pairs of subalgebras of $\mathrm{O}(4 j+2)$. There are two such pairs and their representations have valuable duality relations as discovered by Helmers [3].

Two subalgebras $\mathfrak{g}_{1}$ and $\mathfrak{g}_{2}$ of a Lie algebra $\mathfrak{g}$ are said to be dual within a representation of $\mathfrak{g}$ if $\mathfrak{g}_{1}$ and $\mathfrak{g}_{2}$ are mutual centralizers in $\mathfrak{g}$ and if each set of equivalent irreps of $\mathfrak{g}_{1}$ together comprise an irrep of the direct sum algebra $\mathfrak{g}_{1}$ $\oplus \mathfrak{g}_{2} \subset \mathfrak{g}$ and vice versa $[6,7]$. In less technical terms, this means (i) that $\mathfrak{g}_{1}$ is the maximal subalgebra of all elements of $\mathfrak{g}$ that commute with all elements of $\mathfrak{g}_{2}$ and vice versa, and (ii) that every irrep of $\mathfrak{g}_{1}$ is associated uniquely with a single irrep of $\mathfrak{g}_{2}$, and vice versa, as illustrated in the introduction.

Not every subalgebra of $\mathrm{O}(4 j+2)$ has a dual. However, the two important subalgebras $\mathrm{U}(2 j+1)$ and $\mathrm{SU}(2)_{S}$ do. The dual of $\mathrm{U}(2 j+1)$ is the subalgebra $\mathrm{U}(1)_{S}$ of $\mathrm{SU}(2)_{S}$ spanned by the operator $\hat{S}_{0}$ and the dual of $\mathrm{SU}(2)_{S}$ is the $\mathrm{USp}(2 j+1)$ algebra spanned by the operators $\left\{\hat{C}_{L M} ; \operatorname{Lodd}\right\}$.

The algebra $\mathrm{U}(2 j+1)$ is the maximal subalgebra of number conserving operators in $\mathrm{O}(4 j+2)$. Conversely, any operator in $\mathrm{O}(4 j+2)$ that commutes with all the operators of $\mathrm{U}(2 j+1)$ is proportional to $\hat{S}_{0}$. More importantly, the $\mathrm{U}(2 j+1) \sim \mathrm{U}(1)_{S}$ duality requires that all states of a $\mathrm{U}(2 j$ +1 ) irrep occurring within the totally antisymmetric space of identical nucleons in a single $j$ shell are eigenstates with a common eigenvalue of $\hat{S}_{0}$. This is understood by writing

$$
\hat{S}_{0}=\frac{1}{2}(\hat{n}-\Omega),
$$

where $\hat{n}$ is the number operator. The $\mathrm{U}(2 j+1) \sim \mathrm{U}(1)_{S}$ duality then follows from the fact that all totally antisymmetric $n$ particle states in a single $j$ shell span a $\mathrm{U}(2 j+1)$ irrep.

The $\mathrm{USp}(2 j+1) \sim \mathrm{SU}(2)_{S}$ duality can be inferred from the fact that these two commuting algebras have a complete set of unique extremal (highest and lowest weight) states in common. For example, the state $|n, \mathrm{HW}\rangle$, constructed by putting a nucleon in each of the orbitals with $m=j, j$ $-1, \ldots, j+1-n$, is a $\operatorname{USp}(2 j+1)$ highest weight state and, being annihilated by $\hat{S}_{-}$, it is simultaneously an $\mathrm{SU}(2)_{S}$ lowest weight state,. Such an $n$-particle state contain no zerocoupled pairs and is said to have seniority $v=n$ and quasispin

$$
S=(\Omega-v) / 2
$$

It follows that these states simultaneously define related irreps of $\mathrm{USp}(2 j+1)$ and $\mathrm{SU}(2)_{S}$.

As shown by Helmers [3] in a more general context, $\mathrm{USp}(2 j+1) \sim \mathrm{SU}(2)_{S}$ duality can also be inferred from a linear relationship between the Casimir invariants of these two algebras. The Casimir invariant of $\operatorname{USp}(2 j+1)$ is

$$
\hat{C}_{2}[\operatorname{USp}(2 j+1)]=-2 \sum_{L}^{\text {odd }}[L]\left[\hat{C}_{L} \otimes \hat{C}_{L}\right]_{0} .
$$

It is known from Lie algebra structure theory [13] that all states of a USp $(2 j+1)$ irrep with highest weight $\left(f_{1}, f_{2}, \ldots, f_{j+1 / 2}\right)$ are eigenstates of the Casimir operator with eigenvalues

$$
\mathcal{C}_{2}[\operatorname{USp}(2 j+1)]=\sum_{r=1}^{j+1 / 2} f_{r}\left(f_{r}+2 j+3-2 r\right) .
$$

The irreps of seniority $v$ for a nucleon of a single type in a $j$ shell have highest weights with $f_{r}=1$ for $r \leqslant v$ and $f_{r}=0$ for $r>v$. Thus, in terms of seniority

$$
\mathcal{C}_{2}[\operatorname{USp}(2 j+1)]=v(2 j+3-v)
$$

and, in terms of quasispin,

$$
\mathcal{C}_{2}[\operatorname{USp}(2 j+1)]=\Omega(\Omega+1)-4 S(S+1) .
$$

The $\operatorname{USp}(2 j+1) \sim \operatorname{SU}(2)_{S}$ duality implies that the set of all $v$-nucleon states $|v \rho J M\rangle$ that are annihilated by the quasispin lowering operator, i.e., $\hat{S}_{-}|v \rho J M\rangle=0$, span a $\mathrm{USp}(2 j+1)$ irrep. Moreover, the set of all $n$-nucleon states $|n v \rho J M\rangle \propto\left(\hat{S}_{+}\right)^{(n-v) / 2}|v \rho J M\rangle$ obtained by adding (n $-v) / 2$ zero-spin pairs to $v$-particle quasispin vacuum states span an equivalent $\operatorname{USp}(2 j+1)$ irrep.

Basis states $\{|n v \rho J M\rangle\}$ for a nucleus of $n$ neutrons (or $n$ protons) in a single $j$ shell are classified by the quantum numbers of the subalgebra chain

$$
\begin{array}{ccccc}
\mathrm{U}(2 j+1) & \supset \mathrm{USp}(2 j+1) & \supset \mathrm{SU}(2)_{J} \supset \mathrm{U}(1)_{J}, \\
\left\{1^{n}\right\} & v & \rho & J & M
\end{array}
$$

where $\rho$ is multiplicity index and $\mathrm{SU}(2)_{J}$ is the algebra spanned by the angular momentum operators

$$
\hat{J}_{M}=-\sqrt{\frac{j(j+1)(2 j+1)}{3}} \hat{C}_{1 M} .
$$


It follows from the above noted $\mathrm{U}(2 j+1) \sim \mathrm{U}(1)_{S}$ and $\mathrm{USp}(2 j+1) \sim \mathrm{SU}(2)_{S}$ dualities that the same basis states are also labeled equivalently by the subalgebra chain

$$
\begin{aligned}
& \mathrm{SU}(2)_{S} \times \mathrm{SU}(2)_{J} \supset \mathrm{U}(1)_{S} \times \mathrm{U}(1)_{J}, \\
& \begin{array}{llll}
S & J & S_{0} \quad M
\end{array}
\end{aligned}
$$

with $S=\frac{1}{2}(\Omega-v)$ and $S_{0}=\frac{1}{2}(n-\Omega)$, where the multiplicity index $\rho$ is now interpreted as labeling the multiplicity of $\mathrm{SU}(2)_{S} \times \mathrm{SU}(2)_{J}$ irreps that occur.

With basis states $\{|n v \rho J M\rangle\}$ symmetry adapted to both the subalgebra chains (23) and (25), it is possible to exploit the tensorial properties of operators with respect to the algebras of either chain to facilitate calculations. For example, the $\mathrm{SU}(2)_{S} \oplus \mathrm{SU}(2)_{J}$ algebra can be used to classify simultaneously the second quantized operators of a $j$ shell as quasispin and angular momentum tensors [14].

The fermion operators transform into one another as components of a quasispin $S=\frac{1}{2}$ tensor;

$$
\begin{gathered}
{\left[\hat{S}_{+}, a_{j m}\right]=a_{j m}^{\dagger}, \quad\left[\hat{S}_{-}, a_{j m}^{\dagger}\right]=a_{j m},} \\
{\left[\hat{S}_{0}, a_{j m}^{\dagger}\right]=\frac{1}{2} a_{j m}^{\dagger}, \quad\left[\hat{S}_{0}, a_{j m}\right]=-\frac{1}{2} a_{j m} .}
\end{gathered}
$$

For even $J$ and fixed $M$, the three pair operators $\left\{\hat{A}_{J M}, \hat{C}_{J M}, \hat{B}_{J M}\right\}$, are the $(1,0,-1)$ components of a quasispin $S=1$ tensor and the operators $\left\{\hat{C}_{J M} ; J\right.$ odd $\}$ of the $\mathrm{USp}(2 j+1)$ algebra are quasispin scalars [15].

\section{D. $\mathrm{U}(2 j+1) \supset \mathrm{USp}(2 j+1)$ highest weight states}

The state $|n, \mathrm{HW}\rangle=\left|n, v=n, J_{\max }, M=J_{\max }\right\rangle$ constructed by putting a nucleon in each of the orbitals with $m=j, j$ $-1, \ldots, j+1-n$ is a state of $\mathrm{U}(2 j+1), \mathrm{O}(4 j+2)$, and $\mathrm{SU}(2)_{J}$ highest weight, having maximum angular momentum $J_{\max }=n(2 j+1-n) / 2$. It is a totally antisymmetric and multiplicity-free state with $\mathrm{U}(2 j+1)$ weight $\left\{1^{n}\right\}$, defined by specifying the nonzero occupation factors (in this case 1 or 0$)$ of each orbital in the $m=j, j-1, \ldots,+j$ sequence. Other $n$-particle $\mathrm{O}(4 j+2)$ highest weight states of $v \leqslant n$ are given, to within norm factors, by

$$
\left|n v J_{\max } J_{\max }\right\rangle \propto\left(\hat{S}_{+}\right)^{(n-v) / 2}|v, \mathrm{HW}\rangle .
$$

In calculating matrix elements of operators in the $\mathrm{U}(2 j$ $+1)$ and $\mathrm{O}(4 j+2)$ Lie algebras, it is useful to start with the expectation values of these operators in the highest weight states.

The expectation of the operator $\hat{C}_{L 0}$ in a $\mathrm{U}(2 j+1)$ highest weight state is

$$
\left\langle n, \mathrm{HW}\left|\hat{C}_{L 0}\right| n, \mathrm{HW}\right\rangle=\sum_{m}(-1)^{j+m}(j,-m ; j m \mid L 0) \lambda_{m},
$$

where

$$
\lambda_{m}= \begin{cases}+1 / 2, & j-m<n \\ -1 / 2, & j-m \geqslant n .\end{cases}
$$

The sum in Eq. (28) can be carried out explicitly and gives, for example, the following reduced matrix elements of the $\mathrm{USp}(2 j+1)$ operators, which commute with the quasispin operators:

$$
\begin{gathered}
\frac{\left\langle n v J_{\max }\left\|\hat{C}_{3}\right\| n v J_{\max }\right\rangle}{\sqrt{7}} \\
=-\sqrt{\frac{(2 J+4)(2 J+3) \cdots 2 J}{(2 J-1)(2 J-2)}} \\
\quad \times \frac{\left[x^{2}-(5 n-2) x+\left(5 n^{2}-5 n+2\right)\right]}{\sqrt{(x+4) \cdots(x-2)}}, \quad(30) \\
\frac{\left\langle n v J_{\max }\left\|\hat{C}_{5}\right\| n v J_{\max }\right\rangle}{\sqrt{11}} \\
=-\sqrt{\frac{(2 J+6)(2 J+5) \cdots 2 J}{(2 J-1)(2 J-2)(2 J-3)(2 J-4)}} \\
\quad \times \frac{Q_{5}(x, n)}{\sqrt{(x+6) \cdots(x-4)}}, \\
Q_{5}(x, n)=x^{4}-14 n x^{3}+4 x^{3}+56 n^{2} x^{2}-42 n x^{2}+21 x^{2} \\
-84 n^{3} x+112 n^{2} x-112 n x+34 x+42 n^{4}-84 n^{3} \\
+126 n^{2}-84 n+24, \\
\left.-3 n v J_{\max }\left\|\hat{C}_{7}\right\| n v J_{\max }\right\rangle \\
\sqrt{15}=-\sqrt{\frac{(2 J+8)(2 J+7) \cdots 2 J}{(2 J-1) \cdots(2 J-5)(2 J-6)}} \\
+996 n+240), \\
\quad \times \frac{Q_{7}(x, n)}{\sqrt{(x+8)(x+7) \cdots(x-5)(x-6)}}, \\
+3(9 n-2) x^{5}+5\left(45 n^{2}-27 n+17\right) x^{4}
\end{gathered}
$$

where $x=2 j$.

\section{E. Branching rules}

The space of $n$ identical fermions in a $j$ shell is of dimension $(2 j+1) ! /[n !(2 j+1-n) !]$. It is the carrier space for the totally antisymmetric irrep $\left\{1^{n}\right\}$ of the unitary algebra $\mathrm{U}(2 j+1)$.

The highest weight state of $\mathrm{U}(2 j+1)$ in the $n$-fermion space is also a highest weight state for an irrep of the symplectic subalgebra $\operatorname{USp}(2 j+1)$. The latter is labeled by the seniority quantum number $v=n$, and its dimension is 
TABLE II. Angular momentum branching rules for $j=9 / 2$.

\begin{tabular}{lc}
\hline \hline$v$ & $J$ \\
\hline 0 & 0 \\
1 & $9 / 2$ \\
2 & $2,4,6,8$ \\
3 & $3 / 2,5 / 2,7 / 2,9 / 2,11 / 2,13 / 2,15 / 2,17 / 2,21 / 2$ \\
4 & $0,2,3,4^{2}, 5,6^{2}, 7,8,9,10,12$ \\
5 & $1 / 2,5 / 2,7 / 2,9 / 2,11 / 2,13 / 2,15 / 2,17 / 2,19 / 2,25 / 2$ \\
\hline
\end{tabular}

$$
\operatorname{dim}=\left(\begin{array}{c}
2 j+3 \\
v
\end{array}\right) \frac{2 j+3-2 v}{2 j+3} .
$$

The entire $\mathrm{U}(2 j+1)$ antisymmetric irrep space for $n$ identical fermions decomposes into irreps of $\operatorname{USp}(2 j+1)$ with seniorities $v=n, n-2, \ldots 0$ or 1 .

The angular momentum branching rules for a $\operatorname{USp}(2 j$ +1 ) irrep were given by Flowers [5] and Hamermesh [16] for $j \leqslant 7 / 2$. For $j=9 / 2$ and $j=11 / 2$, Tables II and III provide the $\mathrm{SU}(2)_{J} \subset \mathrm{USp}(2 j+1) \subset \mathrm{SU}(2 j+1)$ branching rules. These decompositions were deduced using the " $m$ scheme" and confirmed by a branching rule code [17]. For example, when $n=2$ identical fermions are in the $j=9 / 2$ shell, the space decomposes into a seniority $v=2$ subspace with $J$ $=2,4,6,8$ and a seniority $v=0$ subspace with $J=0$.

\section{SENIORITY-CONSERVING FORCES}

A two-body interaction (assumed generally to be number conserving and rotationally invariant), acting within a $(j)^{n}$ configuration space, has the standard expansion

$$
\hat{V}=-\frac{1}{4} \sum_{J}[J] V^{J}\left(\hat{A}_{J} \otimes \hat{B}_{J}\right)_{0},
$$

where $[J]=\sqrt{2 J+1}$ : the sum is over even angular momentum $J=0,2, \ldots, 2 j-1$, and $V^{J}$ is the $M$-independent twonucleon matrix element

$$
V^{J}=\left\langle j^{2} ; J M|\hat{V}| j^{2} ; J M\right\rangle
$$

TABLE III. Angular momentum branching rules for $j=11 / 2$.

\begin{tabular}{lc}
\hline \hline$v$ & $J$ \\
\hline 0 & 0 \\
1 & $11 / 2$ \\
2 & $2,4,6,8,10$ \\
3 & $3 / 2,5 / 2,7 / 2,(9 / 2)^{2}, 11 / 2,13 / 2,(15 / 2)^{2}$, \\
4 & $0,2^{2}, 3,4^{3}, 5^{2}, 6^{3}, 7^{2}, 8^{3}, 9^{2}, 10^{2}, 11,12^{2}, 13,14,16$ \\
5 & $1 / 2,3 / 2,(5 / 2)^{2},(7 / 2)^{3},(9 / 2)^{2},(11 / 2)^{3},(13 / 2)^{3}$, \\
& $(15 / 2)^{3},(17 / 2)^{3},(19 / 2)^{3},(21 / 2)^{2},(23 / 2)^{2}$, \\
6 & $0,2,3^{2}, 4^{2}, 5,6^{3}, 7^{2}, 8^{2}, 9^{2}, 10^{2}, 11,12^{2}, 13,14,15$, \\
& 18 \\
\hline \hline
\end{tabular}

The conditions that $\hat{V}$ must satisfy to conserve seniority can be derived by expressing the basis $\left(\hat{A}_{J} \otimes \hat{B}_{J}\right)_{0}$ of two-body rotational scalars as a sum of quasispin tensors. The operators

$$
\begin{gathered}
X^{0}(J)=\left(\hat{A}_{J} \otimes \hat{B}_{J}\right)_{0}-\left(\hat{C}_{J} \otimes \hat{C}_{J}\right)_{0}+\left(\hat{B}_{J} \otimes \hat{A}_{J}\right)_{0}, \\
X_{0}^{1}(J)=\left(\hat{A}_{J} \otimes \hat{B}_{J}\right)_{0}-\left(\hat{B}_{J} \otimes A_{J}\right)_{0}, \\
X_{0}^{2}(J)=\left(\hat{A}_{J} \otimes \hat{B}_{J}\right)_{0}+2\left(\hat{C}_{J} \otimes \hat{C}_{J}\right)_{0}+\left(\hat{B}_{J} \otimes \hat{A}_{J}\right)_{0},
\end{gathered}
$$

are components of quasispin tensor operators $X^{S}(J)$ of rank $S$. The operators $X^{S}(J)$ are constructed by coupling of the quasispin vector operator $\left\{\hat{A}_{J M}, \hat{C}_{J M}, \hat{B}_{J M}\right\}$ with itself.

For compactness of notation, denote the basic two-body scalar operators by the symbols

$$
\hat{Z}_{J}=\left(\hat{A}_{J} \otimes \hat{B}_{J}\right)_{0}, \quad J=0,2,4, \ldots .
$$

The explicit relationship between the $\hat{Z}_{J}$ and $X_{0}^{S}(J)$ operators is found by normal ordering the right hand side of Eq. (36) with the help of the identities

$$
\begin{gathered}
\left(\hat{B}_{J} \otimes \hat{A}_{J}\right)_{0}=\hat{Z}_{J}+\frac{[J]}{\Omega}(\hat{n}-\Omega), \\
\left(\hat{C}_{J} \otimes \hat{C}_{J}\right)_{0}=\sum_{\gamma} M_{J \gamma}^{\Omega} \hat{Z}_{\gamma}+\frac{[J]}{2 \Omega} \hat{n}-\delta_{J, 0}\left(\hat{n}-\frac{1}{2} \Omega\right),
\end{gathered}
$$

where

$$
M_{J \gamma}^{\Omega}=M_{\gamma J}^{\Omega}=2[J][\gamma] W(j j j j ; \gamma J)
$$

and $J, \gamma$ run over the even integers from 0 to $(2 j-1)$. The $S=1$ operators $X_{0}^{1}(J)$ are seen to be proportional to one another and related linearly to the number operator,

$$
X_{0}^{1}(J)=\left[\hat{A}_{J}, \hat{B}_{J}\right]_{0}=\frac{[J]}{\Omega}(\Omega-\hat{n}) .
$$

It follows that, to within constants and terms linear in the number operator, the scalar and tensor quasispin operators are related to the $\hat{Z}_{J}$ operators by

$$
\begin{gathered}
X^{0}(J)=-\left(\hat{M}^{\Omega}-2 \hat{I}\right) \hat{Z}_{J}+\cdots, \\
X_{0}^{2}(J)=2\left(\hat{M}^{\Omega}+\hat{I}\right) \hat{Z}_{J}+\cdots,
\end{gathered}
$$

where $\hat{M}^{\Omega}$ is the linear transformation for which

$$
\hat{M}^{\Omega} \hat{Z}_{J}=\sum_{\gamma} \hat{Z}_{\gamma} M_{\gamma J}^{\Omega}
$$

and $\hat{I}$ is the identity operator; $\hat{I} \hat{Z}_{J}=\hat{Z}_{J}$.

Let $W$ denote the linear space spanned by the $\hat{Z}_{J}$ operators and let $W_{0}$ and $W_{2}$ denote subspaces of $W$ spanned, respectively, by the operators $\left\{\left(\hat{M}^{\Omega}-2 \hat{I}\right) \hat{Z}_{J}\right\}$ and $\left\{\left(\hat{M}^{\Omega}+\hat{I}\right) \hat{Z}_{J}\right\}$. Then, $W_{0}$ and $W_{2}$ are subspaces of two-body operators in $W$ 
that are, respectively, quasispin scalars and tensors to within constants and terms linear in the number operator. For convenience, we shall refer to elements of $W_{0}$ and $W_{2}$ as quasiscalar and quasitensor operators, respectively.

Proposition 1. The eigenvalues of $\hat{M}^{\Omega}$ are equal to -1 or 2. The space of operators $W$ is the direct sum

$$
W=W_{0} \oplus W_{2}
$$

and

$$
\hat{P}_{0}=-\frac{1}{3}\left(\hat{M}^{\Omega}-2 \hat{I}\right), \quad \hat{P}_{2}=\frac{1}{3}\left(\hat{M}^{\Omega}+\hat{I}\right),
$$

are, respectively, $W \rightarrow W_{0}$ and $W \rightarrow W_{2}$ projection operators.

Proof. The matrix $M^{\Omega}$ is real and symmetric. Therefore $\hat{M}^{\Omega}$ is completely diagonalizable. Let $\hat{U} \in W$ be an eigenvector of $\hat{M}^{\Omega}$ of eigenvalue $k$. It follows from Eqs. (42) and (43) that

$$
\begin{aligned}
& \left(\hat{M}^{\Omega}-2 \hat{I}\right) \hat{U}=(k-2) \hat{U} \in W_{0}, \\
& \left(\hat{M}^{\Omega}+\hat{I}\right) \hat{U}=(k+1) \hat{U} \in W_{2},
\end{aligned}
$$

and, because $W_{0}$ and $W_{2}$ have no nonzero vectors in common, $k$ must be equal to -1 or 2 . It also follows that $W$ is the direct sum of $W_{0}$ and $W_{2}$ and that

$$
\begin{aligned}
& \hat{P}_{0} \hat{U}=\hat{U} \quad \text { for all } \quad \hat{U} \in W_{0} \\
& \hat{P}_{2} \hat{U}=\hat{U} \quad \text { for all } \quad \hat{U} \in W_{2} .
\end{aligned}
$$

Thus, $\hat{P}_{0}^{2}=\hat{P}_{0}$ and $\hat{P}_{2}^{2}=\hat{P}_{2}$ and $P_{0}: W \rightarrow W_{0}$ and $P_{2}: W$ $\rightarrow W_{2}$ are projection operators.

Let $p_{-1}=\operatorname{dim} W_{0}$ and $p_{2}=\operatorname{dim} W_{2}$ denote the dimensions of the eigenspaces of $\hat{M}^{\Omega}$ belonging to the eigenvalues -1 and 2, respectively. We now give an explicit expression for $p_{2}$ and thereby obtain by our new methods a result given previously by Talmi [2] based on results of de-Shalit and Talmi [10] and Ginocchio and Haxton [11].

Proposition 2 (Talmi). The number of independent rotationally invariant two-body interactions that mix seniority in a $(j)^{n}$ configuration space is equal to $[(2 j-3) / 6]$, where $[x]$ denotes the largest integer less than or equal to $x$.

Proof. As a result of the duality relationship between $\mathrm{USp}(2 j+1)$ and $\mathrm{SU}(2)_{S}$, any two-body interaction that conserves quasispin also conserves seniority. Thus, the set of seniority conserving interactions includes all quasiscalar operators (elements of $W_{0}$ ) and the monopole pairing interaction $-G \hat{S}_{+} \hat{S}_{-}$proportional to $X_{0}^{2}(0) \in W_{2}$. The number of independent two-body interactions that mix seniority is therefore $p_{2}-1$ and, from the first proposition, $p_{2}$ is given by

$$
p_{2}=\operatorname{tr} \hat{P}_{2}=\frac{1}{3}\left(\Omega+2 \sum_{J \text { even }}^{2 j-1}(2 J+1) W(j j j j ; J J)\right)=\left[\frac{2 j+3}{6}\right] .
$$

TABLE IV. The number $p_{-1}\left(p_{2}-1\right)$ of linearly independent rotationally invariant two-body quasispin scalar (seniority-mixing) interactions in a $j$ shell.

\begin{tabular}{lcc}
\hline \hline$j$ & $p_{-1}$ & $p_{2}-1$ \\
\hline $3 / 2$ & 1 & 0 \\
$5 / 2$ & 2 & 0 \\
$7 / 2$ & 3 & 0 \\
$9 / 2$ & 3 & 1 \\
$11 / 2$ & 4 & 1 \\
$13 / 2$ & 5 & 1 \\
$15 / 2$ & 5 & 2 \\
$17 / 2$ & 6 & 2 \\
$19 / 2$ & 7 & 2 \\
$21 / 2$ & 7 & 3 \\
\hline \hline
\end{tabular}

Note that in the last line of Eq. (51) we have used a sum rule for the Racah coefficient that was conjectured and confirmed, using Maple with exact arithmetic, for all $j<260$.

In Table IV the numbers of two-body interactions that conserve and break seniority in a $(j)^{n}$ configuration space are listed. Thus, for a $(j)^{n}$ configuration space, seniority is a good quantum number for a wide range of interactions. For example, in the $j=13 / 2$ shell, there are seven linearly independent two-body interactions, but only one of the seven mixes seniority. Proposition 2 implies that a two-body interaction must satisfy $p_{2}-1$ constraint conditions in order for it to conserve seniority.

Using the results of Proposition 1, we can now derive explicit conditions that an interaction

$$
\hat{V}=-\frac{1}{4} \sum_{J}[J] V^{J} \hat{Z}_{J} \in W
$$

must satisfy to conserve seniority. To be a quasiscalar, $\hat{V}$ must lie in the subspace $W_{0} \subseteq W$; hence, by Proposition 1 , it must satisfy the equation $\left(\hat{M}^{\Omega}+\hat{I}\right) \hat{V}=0$ so that its projection $\hat{P}_{2} \hat{V}$ onto $W_{2}$ should vanish. However, as observed above, the monopole pairing operator, proportional to $\hat{Z}_{0}$ also conserves seniority. Thus, to conserve seniority, it is sufficient that the projection $\hat{P}_{2} \hat{V}$ of $\hat{V}$ onto the subspace $W_{2} \subset W$ should be proportional to the component $\hat{P}_{2} \hat{Z}_{0} \in W_{2}$ of $\hat{Z}_{0}$. In other words, $\hat{V}$ should satisfy the equation

$$
\left(\hat{M}^{\Omega}+\hat{I}\right) \hat{V}=\lambda\left(\hat{M}^{\Omega}+\hat{I}\right) \hat{Z}_{0}
$$

for some $\lambda$. The coefficients $V^{J}$ of the interaction, should then satisfy the equations

$$
[J] V^{J}+\sum_{\gamma}[\gamma] M_{J \gamma}^{\Omega} V^{\gamma}=\lambda\left(\delta_{J 0}+M_{J 0}^{\Omega}\right) .
$$

for all even values of $J$ and some value of $\lambda$.

The value of $\lambda$, determined for a given interaction from the $J=0$ equation, is

$$
\lambda=V^{0}-\frac{2}{2 j-1} \sum_{\gamma>0}(2 \gamma+1) V^{\gamma} .
$$


Eliminating $\lambda$ from the $J \geqslant 2$ equations and making use of the identity [2]

$$
2 \sum_{\gamma}^{\text {even }}(2 \gamma+1) W(j j j j: J \gamma)=1-(2 j+1) \delta_{J 0},
$$

then leads to Talmi's theorem [2]

Theorem 1 (Talmi). A necessary and sufficient set of conditions for the $V^{J}$ coefficients of a two-body interaction to conserve seniority are that

$$
V^{J}-\Delta+2 \sum_{\gamma}^{\text {even }}(2 \gamma+1) W(j j j j: J \gamma)\left(V^{\gamma}-\Delta\right)=0,
$$

for all (even) values of $J \geqslant 2$, where

$$
\Delta=\frac{1}{(j+1)(2 j-1)} \sum_{\gamma>0}(2 \gamma+1) V^{\gamma} .
$$

From Proposition 2, it now follows that among the set of $(2 j-1) / 2=\Omega-1$ conditions given by this theorem, only $[(\Omega+1) / 3]$ equations are linearly independent. For $j \leqslant 7 / 2$, all interactions conserve seniority and, for $9 / 2 \leqslant j \leqslant 13 / 2$, there is a single condition

$$
\begin{gathered}
65 V^{2}-315 V^{4}+403 V^{6}-153 V^{8}=0 \text { for } j=\frac{9}{2} \\
1020 V^{2}-3519 V^{4}+637 V^{6}+4403 V^{8}-2541 V^{10}=0 \\
\text { for } j=\frac{11}{2}, \\
1615 V^{2}-4275 V^{4}-1456 V^{6}+3196 V^{8}+5145 V^{10}-4225 V^{12} \\
=0 \quad \text { for } j=\frac{13}{2} .
\end{gathered}
$$

The duality relationship between the $\operatorname{USp}(2 j+1)$ and $\mathrm{SU}(2)_{S}$ quasispin algebras implies that any two-body interaction that conserves seniority is a quadratic in the elements of the $\mathrm{USp}(2 j+1)$ and $\mathrm{U}(2)_{S}$ algebras. This result follows directly from Burnside's theorem [18] which states that:

Any linear operator on the Hilbert space $\mathbb{H}_{0}$ for an irreducible representation $T$ of a Lie algebra $\mathfrak{g}$ is an element of the associative algebra of linear operators on $\mathbb{H}_{0}$ generated by the operators $\{T(X) ; X \in \mathfrak{g}\}$.

In other words, if the elements $\left\{X_{i}\right\}$ of a basis for $\mathfrak{g}$ have an irreducible representation as linear operators $\left\{\hat{X}_{i}\right\}$ on a Hilbert space $\mathbb{H}_{0}$, then any linear operator $H$, such as a Hamiltonian, which leaves $\mathbb{H}_{0}$ invariant is expressible in the form

$$
H=c_{0}+\sum_{i} c_{i} \hat{X}_{i}+\sum_{i j} c_{i j} \hat{X}_{i} \hat{X}_{j}+\cdots,
$$

where $c_{0}, c_{i}, c_{i j}$, etc., are complex coefficients. Thus, we obtain the theorem:

Theorem 2 (Talmi). Any rotationally invariant numberconserving hermitian Hamiltonian with seniority-conserving two-body interactions acting within a single $j$ shell can be expressed in the form

$$
H=\varepsilon \hat{n}-G \hat{S}_{+} \hat{S}_{-}+V_{0},
$$

where $V_{0}$ is a linear combination of the $\left\{\left[\hat{C}_{L} \otimes \hat{C}_{L}\right]_{0} ; L\right.$ $=1,3,5, \ldots, 2 j\}$ operators.

The classification of states $\{|n v \rho J M\rangle\}$ by the subalgebra chain (23) also implies that a two-body interaction that conserves seniority should be of the form of $V_{0}$ plus a term quadratic in the number operator. The latter result is consistent with Talmi's theorem because, to within terms quadratic in the number operator, the operator $\hat{S}_{+} \hat{S}_{-}$is the $\mathrm{SU}(2)_{S}$ Casimir invariant which, by the duality relationship (22), is linearly related to the $\operatorname{USp}(2 j+1)$ Casimir invariant (19) which, in turn, is of the form required for $V_{0}$.

Given the form of any seniority-conserving interaction (Theorem 2) and the number of independent quasispin scalars two-body interactions (Proposition 2), it is possible to construct a basis of seniority conserving interactions explicitly. The number $p_{-1}$ of linearly-independent quasispin scalars for $j=\frac{7}{2}$ and $j=\frac{9}{2}$ is equal to three. Two of these scalars are $\hat{\mathcal{C}}_{2}[\mathrm{USp}(2 j+1)]$ and $\hat{J} \cdot \hat{J}$. A convenient choice for the third scalar is $\left[\hat{C}_{3} \otimes \hat{C}_{3}\right]_{0}$. For $j=\frac{11}{2}$, the seniorityconserving interaction consists of four operators. The fourth operator can be $\left[\hat{C}_{5} \otimes \hat{C}_{5}\right]_{0}$, for example. For $j=\frac{13}{2}$ and $j$ $=\frac{15}{2}$, the seniority-conserving interaction consists of five operators, and so on. The seniority conserving Hamiltonian (63) for $j=9 / 2$, for example, can then be expressed as

$$
H=H_{0}+a \mathbf{J}^{2}+b \hat{\mathcal{C}}_{2}[\mathrm{USp}(10)]+c \hat{Z},
$$

where $H_{0}$ depends only on the particle number and $\hat{Z}$ $=-\left(\hat{C}_{3} \otimes \hat{C}_{3}\right)_{0} / \sqrt{7}$. The energies of this Hamiltonian are then

$$
E_{n \rho v J}=E_{n 0}+a J(J+1)+b v(2 j+3-v)+c Z_{\rho v J} .
$$

where $Z_{\rho v J}$ is an eigenvalue of $\hat{Z}$. The excitation energies are independent of $n$.

\section{MATRIX ELEMENTS OF LIE ALGEBRAS}

This section presents a method for computing reduced matrix elements of the $\left\{\hat{C}_{L} ; \operatorname{Lodd}\right\}$ operators in the $\operatorname{USp}(2 j$ $+1)$ Lie algebra. The desired matrix elements are shown to be solutions of a sequence of equations. The early equations in the set involve a small number of unknown matrix elements and some of these equations can be solved analytically for the unknowns. Subsequent sets of equations involve more unknowns and it rapidly becomes impractical (even impossible, in general) to solve all of them analytically. It is nevertheless possible, as we show, to solve them numerically.

Once these matrix elements are determined, the matrix elements of quasispin scalar operators $\left(\hat{C}_{L} \otimes \hat{C}_{L}\right)_{0}$, which are possible two-body interaction terms of a general seniorityconserving Hamiltonian, are easily inferred. Analytic formulas are reported for some of the energy eigenvalues of quasispin scalar Hamiltonians. An eigenstate of a seniorityconserving interaction that has an analytic expression for its energy is said to possess a $\operatorname{USp}(2 j+1)$ partial dynamical 
symmetry according to Leviatan's definition [1].

Let $|v \rho J M\rangle$ denote an orthonormal basis for the irreducible representation of $\operatorname{USp}(2 j+1)$ in a $j$ shell with seniority $v$, where $\rho$ is the multiplicity index for the states with total angular momentum $J$ and $z$-component $M$. The branching rules of Sec. II $E$ determine the range of quantum numbers in this basis. The problem is to find the reduced matrix elements of the unitary symplectic algebra generators, $\left\langle v \rho^{\prime} J^{\prime}\left\|\hat{C}_{L}\right\| v \rho J\right\rangle$ for odd $L$. These matrix elements are solutions to the equations

$$
\begin{aligned}
(\eta-1) & W\left(L_{1} j L_{2} j ; j L\right) \frac{\left\langle v \rho^{\prime} J^{\prime}\left\|\hat{C}_{L}\right\| v \rho J\right\rangle}{\sqrt{2 L+1}} \\
= & \sum_{\rho^{\prime \prime} J^{\prime \prime}}\left\{W\left(J L_{2} J^{\prime} L_{1} ; J^{\prime \prime} L\right) \frac{\left\langle v \rho^{\prime} J^{\prime}\left\|\hat{C}_{L_{1}}\right\| v \rho^{\prime \prime} J^{\prime \prime}\right\rangle}{\sqrt{2 L_{1}+1}}\right. \\
& \times \frac{\left\langle v \rho^{\prime \prime} J^{\prime \prime}\left\|\hat{C}_{L_{2}}\right\| v \rho J\right\rangle}{\sqrt{2 L_{2}+1}}-\eta W\left(J L_{1} J^{\prime} L_{2} ; J^{\prime \prime} L\right) \\
& \left.\times \frac{\left\langle v \rho^{\prime} J^{\prime}\left\|\hat{C}_{L_{2}}\right\| v \rho^{\prime \prime} J^{\prime \prime}\right\rangle}{\sqrt{2 L_{2}+1}} \frac{\left\langle v \rho^{\prime \prime} J^{\prime \prime}\left\|\hat{C}_{L_{1}}\right\| v \rho J\right\rangle}{\sqrt{2 L_{1}+1}}\right\},
\end{aligned}
$$

where $\eta=(-1)^{L_{1}+L_{2}-L}$, derived from the commutation relations (13). For a unitary representation, the $\hat{C}_{L}$ operators must also satisfy a hermiticity condition,

$$
\left\langle v \rho^{\prime} J^{\prime}\left\|\hat{C}_{L}\right\| v \rho J\right\rangle^{*}=(-1)^{J-J^{\prime}}\left\langle v \rho J\left\|\hat{C}_{L}\right\| v \rho^{\prime} J^{\prime}\right\rangle .
$$

\section{A. Recursion formulas}

Expectation values of the $\mathrm{USp}(2 j+1)$ operators with respect to the irrep's highest weight vector are known, see Eq. (28). Starting with these values, matrix elements with respect to other basis states can be calculated sequentially. Consider, for example, the special cases of Eq. (66) with $L=1,3, L_{1}$ $=L_{2}=3$, and $\left|v \rho^{\prime} J^{\prime}\right\rangle=|v \rho J\rangle=|v, \mathrm{HW}\rangle$. Angular momentum coupling rules and the irrep branching rules limit the intermediate state sums in Eq. (66) for these cases to just two terms. Matrix elements of the $L=1$ operators $\hat{C}_{1}$, being proportional to angular momentum operators are already known. By applying the highest weight data (30) and the hermiticity condition (67), the following solutions for the other unknown matrix elements are found:

$$
\begin{gathered}
\frac{\left|\left\langle v(J-2)\left\|\hat{C}_{3}\right\| v J\right\rangle\right|^{2}}{7}=\frac{(2 J+2)(2 J+1) 2 J}{(x+4)(x+3) \cdots(x-2)} \frac{60\left(n^{2}-1\right)(x-2 n+1)^{2}(x-n)(x-n+2)}{(2 J-4)(2 J-1)}, \\
\frac{\left|\left\langle v(J-3)\left\|\hat{C}_{3}\right\| v J\right\rangle\right|^{2}}{7}=\frac{(2 J+2)(2 J+1) 2 J}{(x+4)(x+3) \cdots(x-2)} \frac{60\left(n^{2}-1\right)\left(n^{2}-4\right)(x-n-1)(x-n)(x-n+2)(x-n+3)}{(2 J-4)(2 J-2)(2 J+2)},
\end{gathered}
$$

where $x=2 j$ and $|v J\rangle=|v, \mathrm{HW}\rangle$ denotes the multiplicity free state of $J=J_{\max }$. We take the real positive square root for these two reduced matrix elements as our phase choice.

Similarly, for the $L=3,5$ special cases with $L_{1}=3, L_{2}=5$, we obtain the solution

$$
\begin{aligned}
\left\langle v(J-2)\left\|\hat{C}_{5}\right\| v J\right\rangle= & \sqrt{\frac{4620(2 J+4)(2 J+3)(2 J+2)(2 J+1) 2 J}{(2 J-5)(2 J-6)(2 J-4)(2 J-1)}}(x-2 n+1)\left(x^{2}-12 J+2 x+12\right) \\
\times & \sqrt{\frac{\left(n^{2}-1\right)(x-n)(x-n+2)}{(x+6)(x+5) \cdots(x-4)}} \\
\left\langle v(J-3)\left\|\hat{C}_{5}\right\| v J\right\rangle= & \sqrt{\frac{4620(2 J+3)(2 J+2)(2 J+1) 2 J}{(2 J-7)(2 J-6)(2 J-4)(2 J-2)}}\left(2 x^{2}-18 J+4 x+12\right) \\
& \times \sqrt{\frac{\left(n^{2}-1\right)\left(n^{2}-4\right)(x-n-1)(x-n)(x-n+2)(x-n+3)}{(x+6)(x+5) \cdots(x-4)}}
\end{aligned}
$$

again with $J=J_{\max }$ and $x=2 j$.

More reduced matrix elements can be evaluated by stepping down from the highest weight state. For example, putting $L_{1}=L_{2}=3$, and $L=1,3,5$ into Eq. (66) with $\left|v \rho^{\prime} J^{\prime}\right\rangle=\left|v\left(J_{\max }-2\right)\right\rangle$ and $|v \rho J\rangle=\left|v J_{\max }\right\rangle$ yields three equations for three unknown matrix elements with the solution 


$$
\begin{aligned}
& \left\langle v(J-2)\left\|\hat{C}_{3}\right\| v(J-2)\right\rangle=-\sqrt{\frac{14 J(2 J-3)(2 J-2)}{(2 J-6)(2 J-5)(2 J-4)(2 J-1)}} \frac{Q_{232}}{\sqrt{(x+4)(x+3) \cdots(x-2)}}, \\
& Q_{232}=n^{2} x^{4}-7 n^{3} x^{3}+4 n^{2} x^{3}-25 n x^{3}+16 n^{4} x^{2}-21 n^{3} x^{2}+157 n^{2} x^{2} \\
& -75 n x^{2}-36 x^{2}-15 n^{5} x+32 n^{4} x-273 n^{3} x+306 n^{2} x-40 n x \\
& -72 x+5 n^{6}-15 n^{5}+142 n^{4}-259 n^{3}+117 n^{2}+10 n+48, \\
& \left\langle v(J-3)\left\|\hat{C}_{3}\right\| v(J-3)\right\rangle=-\sqrt{\frac{7(2 J-5)(2 J-3)}{(2 J-8)(2 J-7)(2 J-6)(2 J-4)(2 J-2)}} \frac{Q_{333}}{\sqrt{(x+4)(x+3) \cdots(x-2)}}, \\
& Q_{333}=n^{3} x^{5}-8 n^{4} x^{4}+5 n^{3} x^{4}-42 n^{2} x^{4}+23 n^{5} x^{3}-32 n^{4} x^{3}+305 n^{3} x^{3} \\
& -168 n^{2} x^{3}-136 n x^{3}-31 n^{6} x^{2}+69 n^{5} x^{2}-723 n^{4} x^{2}+895 n^{3} x^{2} \\
& -78 n^{2} x^{2}-408 n x^{2}+192 x^{2}+20 n^{7} x-62 n^{6} x+702 n^{5} x-1382 n^{4} x \\
& +814 n^{3} x+180 n^{2} x+656 n x+384 x-5 n^{8}+20 n^{7}-242 n^{6}+656 n^{5} \\
& -645 n^{4}+220 n^{3}-932 n^{2}+928 n-1536, \\
& \left\langle v(J-2)\left\|\hat{C}_{3}\right\| v(J-3)\right\rangle=-30 \sqrt{14}(2 J)(x-2 n+1) \sqrt{\frac{(2 J-3)}{(2 J-4)(2 J-6)(2 J-7)}} \sqrt{\frac{\left(n^{2}-4\right)(x-n-1)(x-n+3)}{(x+4)(x+3) \cdots(x-2)}},
\end{aligned}
$$

with $J=J_{\max }$ and $x=2 j$.

Some additional $\operatorname{USp}(2 j+1)$ reduced matrix elements obtained by sequential solution of the equations are

$$
\begin{aligned}
& \sum_{\rho}\left|\left\langle v(J-2)\left\|\hat{C}_{3}\right\| v \rho(J-4)\right\rangle\right|^{2}=\frac{840(2 J-1)(2 J-2)}{(2 J-8)(2 J-6)(2 J-5)} \frac{Q_{234}}{(x+4)(x+3) \cdots(x-2)}, \\
& Q_{234}= n^{4} x^{6}+n^{2} x^{6}-8 n^{5} x^{5}+6 n^{4} x^{5}-44 n^{3} x^{5}+6 n^{2} x^{5}+24 n x^{5}+26 n^{6} x^{4}-40 n^{5} x^{4} \\
&+302 n^{4} x^{4}-220 n^{3} x^{4}+20 n^{2} x^{4}+120 n x^{4}+144 x^{4}-44 n^{7} x^{3}+104 n^{6} x^{3} \\
&-846 n^{5} x^{3}+1168 n^{4} x^{3}-1174 n^{3} x^{3}+40 n^{2} x^{3}-972 n x^{3}+576 x^{3}+41 n^{8} x^{2} \\
&-132 n^{7} x^{2}+1158 n^{6} x^{2}-2378 n^{5} x^{2}+3794 n^{4} x^{2}-2642 n^{3} x^{2}+5169 n^{2} x^{2} \\
&-3396 n x^{2}-1008 x^{2}-20 n^{9} x+82 n^{8} x-772 n^{7} x+2108 n^{6} x-4366 n^{5} x \\
&+5268 n^{4} x-10246 n^{3} x+10274 n^{2} x-492 n x-3168 x+4 n^{10}+20 n^{9}+201 n^{8} \\
&-684 n^{7}+1704 n^{6}-2802 n^{5}+6257 n^{4}-8602 n^{3}+2106 n^{2}+1836 n+3456, \\
& \sum_{\rho} \mid\langle v(J-2) \|\left.\hat{C}_{3} \| v \rho(J-5)\right\rangle\left.\right|^{2}=\frac{(2 J)(2 J-1)(2 J-3)}{(2 J-8)(2 J-7)(2 J-6)(2 J-4)} \frac{420\left(n^{2}-4\right)(x-n-1)(x-n+3) Q_{235}}{(x+4)(x+3) \cdots(x-2)}, \\
& Q_{235}= n^{3} x^{3}+5 n x^{3}-3 n^{4} x^{2}+3 n^{3} x^{2}-57 n^{2} x^{2}+15 n x^{2}+3 n^{5} x-6 n^{4} x \\
&+107 n^{3} x-114 n^{2} x+334 n x-n^{6}+3 n^{5}-55 n^{4}+105 n^{3}-376 n^{2}+324 n-1008, \\
&\left\langle v(J-2)\left\|\hat{C}_{5}\right\| v J\right\rangle= \sqrt{\frac{4620(2 J+4)(2 J+3)(2 J+2)(2 J+1) 2 J}{(2 J-5)(2 J-6)(2 J-4)(2 J-1)}}(x-2 n+1)\left(x^{2}-12 J+2 x+12\right) \\
& \times \sqrt{\frac{\left(n^{2}-1\right)(x-n)(x-n+2)}{(x+6)(x+5) \cdots(x-4)},}
\end{aligned}
$$




$$
\begin{aligned}
\left\langle v(J-3)\left\|\hat{C}_{5}\right\| v J\right\rangle= & \sqrt{\frac{4620(2 J+3)(2 J+2)(2 J+1) 2 J}{(2 J-7)(2 J-6)(2 J-4)(2 J-2)}}\left(2 x^{2}-18 J+4 x+12\right) \\
& \times \sqrt{\frac{\left(n^{2}-1\right)\left(n^{2}-4\right)(x-n-1)(x-n)(x-n+2)(x-n+3)}{(x+6)(x+5) \cdots(x-4)}} .
\end{aligned}
$$

For any seniority $v$, the $\hat{Z}$ eigenvalues for angular momentum $J=J_{\max }=v(x-v+1) / 2$ and $J=J_{\max }-2$ are deduced using the formulas of this section for the matrix elements of $C_{3}$,

$$
\begin{aligned}
&\left\langle v J_{\max }|\hat{Z}| v J_{\max }\right\rangle=\frac{v(x-v+1)}{(x+4) \cdots(x-2)}\left(v x^{5}-11 v^{2} x^{4}+5 v x^{4}+12 x^{4}+45 v^{3} x^{3}-44 v^{2} x^{3}-48 v x^{3}\right. \\
&+ 48 x^{3}-85 v^{4} x^{2}+135 v^{3} x^{2}+102 v^{2} x^{2}-164 v x^{2}-24 x^{2}+75 v^{5} x-170 v^{4} x-85 v^{3} x \\
&+\left.292 v^{2} x+12 v x-144 x-25 v^{6}+75 v^{5}+25 v^{4}-175 v^{3}-24 v^{2}+124 v+48\right), \\
&\left\langle v\left(J_{\max }-2\right)|\hat{Z}| v\left(J_{\max }-2\right)\right\rangle= \frac{1}{(x+4) \cdots(x-2)}\left(v^{2} x^{6}-12 v^{3} x^{5}+6 v^{2} x^{5}-36 v x^{5}+56 v^{4} x^{4}-60 v^{3} x^{4}\right. \\
&+593 v^{2} x^{4}-180 v x^{4}+48 x^{4}-130 v^{5} x^{3}+224 v^{4} x^{3}-2774 v^{3} x^{3}+2332 v^{2} x^{3} \\
&-432 v x^{3}+192 x^{3}+160 v^{6} x^{2}-390 v^{5} x^{2}+5588 v^{4} x^{2}-8082 v^{3} x^{2} \\
&+3376 v^{2} x^{2}-576 v x^{2}-336 x^{2}-100 v^{7} x+320 v^{6} x-5100 v^{5} x+10728 v^{4} x \\
&-7664 v^{3} x+2104 v^{2} x+48 v x-1056 x+25 v^{8}-100 v^{7}+1730 v^{6}-4840 v^{5} \\
&\left.+5129 v^{4}-2308 v^{3}+28 v^{2}+336 v+1152\right) .
\end{aligned}
$$

\section{B. Numerical solutions}

When the dimensions of a $\operatorname{USp}(2 j+1)$ irrep are large, and especially when there are multiple basis states of the same angular momentum, the above analytical solution of all the equations becomes prohibitively difficult and ultimately impossible. The equations can nevertheless be solved numerically for quite large irreps.

Let $X$ denote a column vector whose entries are the unknown reduced matrix elements of the $\operatorname{USp}(2 j+1)$ operators. Thus, $X$ excludes any known or already determined matrix elements. Moreover, $X$ includes only one of each pair of unknown matrix elements, $\left\langle v \rho^{\prime} J^{\prime}\left\|\hat{C}_{L}\right\| v \rho J\right\rangle$ and $\left\langle v \rho J\left\|\hat{C}_{L}\right\| v \rho^{\prime} J^{\prime}\right\rangle$, since these are not independent for a unitary representation.

The commutation relations (66) determine a set of equations for $X$. The number of independent equations grows rapidly with the seniority $v$ and the shell's dimension $(2 j$ $+1)$. For two particles in the $j=11 / 2$ shell, there are 47 unknown reduced matrix elements that satisfy 1275 distinct equations. For four particles in the $j=9 / 2(j=11 / 2)$ shell there are 243 (1281) unknown reduced matrix elements that satisfy $4550(32,130)$ distinct equations.

Each equation that the unknown matrix elements satisfy can be expressed in the form $f_{\alpha}(X)=0$, where $\alpha$ indexes the set of distinct equations. All the equations will be satisfied simultaneously when $F(X)=\Sigma_{\alpha}\left[f_{\alpha}(X)\right]^{2}$ is zero.

A solution to $F(X)=0$ also minimizes the nonnegative function $F(X)$ and, hence, the derivative $\nabla F(X)$ vanishes at a solution $X$. To find the relative minima of $F(X)$, the method of steepest descent is used in our calculations. Suppose $X_{0}$ is an initial guess for $X$. The linearization of $F(X)$ about $X_{0}$ gives a first approximation $X_{1}$ for $X$ by

$$
0=F\left(X_{0}\right)+\nabla F\left(X_{0}\right) \cdot\left(X_{1}-X_{0}\right) \approx F\left(X_{1}\right) .
$$

The zero of the linearized function, and, therefore, the approximation to the zero of the function is

$$
X_{1}=X_{0}+\epsilon \nabla F\left(X_{0}\right)
$$

with $\epsilon=-F\left(X_{0}\right) /\left|\nabla F\left(X_{0}\right)\right|^{2}$.

If $F\left(X_{1}\right)$ were to vanish, then a solution is found. Otherwise, $X_{1}$ is used as the next (and one hopes better) guess for the starting point of the method of steepest descent. By iterating the steepest descent algorithm, a sequence of points $X_{0}, X_{1}, X_{2}, \ldots$ is computed that, in favorable cases, converges to a relative minimum of the function $F$.

In shells with $j \leqslant 11 / 2$ and all possible particle numbers $n$, computer calculations using the method of steepest descent converged after about 100 iterations to an approximate solution, $F(X)<10^{-22}$. Without exception, the steepest descent method converged to a global minium and a true solution to the commutation relations.

Convergence to a relative minimum is expected when using the steepest descent method. But convergence to a global minimum is unusual. The inference is that the function $F(X)$ 
TABLE V. Energy levels in $\mathrm{keV}$ of ${ }^{92}$ Mo positive parity states. The theoretical levels are calculated in the $\mathrm{USp}(10)$ approximation.

\begin{tabular}{lccc}
\hline \hline \multicolumn{2}{c}{ USp(10) Theory } \\
$J$ & $v=0$ & $v=2$ & Experiment \\
\hline 0 & 0 & & 0 \\
2 & & 1509 & 1509 \\
4 & 2283 & 2283 \\
6 & 2589 & 2612 \\
8 & 2761 & 2761 \\
\hline \hline
\end{tabular}

is very special; in a large neighborhood containing the global minimum, $F$ has no relative minimum besides the global one.

It is worth noting that, if the dimension of $X$ and the number of equations are not excessive, then it is possible to solve for all the unknown matrix elements simultaneously. More generally, it is possible to proceed sequentially, as described in the previous subsection, to obtain solutions for subsets of of matrix elements in which the number of unknown matrix elements at each step is kept much below the total number of unknowns.

\section{Analytic formulas redux}

The reduced matrix elements of $\operatorname{USp}(2 j+1)$ operators are frequently the square roots of rational numbers, cf. Sec. IV A. This favorable situation for a matrix element occurs routinely when the angular momentum is multiplicity free for both the bra and ket vectors. The quantum system is then said to exhibit a partial dynamical symmetry (PDS) among the multiplicity free state vectors.

Using double precision arithmetic in Fortran, the square of a reduced matrix element can be expressed as a rational number with an error that is less than $10^{-15}$. By calculating these rational numbers among a number of PDS states, patterns can be recognized that suggest analytic formulas [20].

For example, when $j \leqslant 21 / 2$ and for seniority $v=2$, the difference between the numerically-calculated matrix elements of the scalar operator $\hat{Z}=-\left[\hat{C}_{3} \times C_{3}\right]_{0} / \sqrt{7}$ and the following formula is less than $10^{-21}$ :

$$
\begin{aligned}
\langle 2 J|\hat{Z}| 2 J\rangle= & \frac{4 J(J+1)}{(x+4) \cdots(x-2)}\left\{6 x^{4}+24 x^{3}-3[5 J(J+1)\right. \\
& +4] x^{2}-6[5 J(J+1)+12] x+2\left[5 J^{2}(J+1)^{2}\right. \\
& +20 J(J+1)+12]\}
\end{aligned}
$$

where $x=2 j$, and the angular momentum $J=x-1, x$ $-3, \ldots, 2$.

\section{APPLICATIONS}

We apply the seniority-conserving model to the low lying energy levels of the $N=50$ isotones, ${ }^{92} \mathrm{Mo},{ }^{94} \mathrm{Ru}$, and ${ }^{96} \mathrm{Pd}$, considered as systems of a closed neutron shell plus active protons in the $g_{9 / 2}$ shell. The experimental energies of the $J$ $=2,4,8$ yrast levels determine the three parameters $(a, b, c)$
TABLE VI. Hamiltonian parameters in $\mathrm{keV}$ of seniorityconserving interactions.

\begin{tabular}{lrrr}
\hline \hline Constant & \multicolumn{1}{c}{${ }^{92} \mathrm{Mo}$} & \multicolumn{1}{c}{${ }^{94} \mathrm{Ru}$} & \multicolumn{1}{c}{${ }^{96} \mathrm{Pd}$} \\
\hline $\mathrm{a}$ & 20.36 & 19.74 & 18.13 \\
$\mathrm{~b}$ & -1227.38 & -1203.90 & -1079.76 \\
$\mathrm{c}$ & -96.37 & -89.75 & -93.40 \\
\hline \hline
\end{tabular}

of the Hamiltonian (64). Equation (65) then predicts the energy of the $J=6$ state. Equivalently, one could say that, for a seniority-conserving interaction, Eq. (59) gives directly the energy of the $J=6$ state in terms of the experimental energies of the $J=2,4,8$ states. Table $\mathrm{V}$ shows that seniority is an approximate symmetry for these energy levels of ${ }^{92}$ Mo since the experimental energy of its $J=6$ level is just $23 \mathrm{keV}$ above the value required for conservation of seniority. The $j=9 / 2$ shell spaces of the ${ }^{94} \mathrm{Ru}$ ( $n=4$ protons) and ${ }^{96} \mathrm{Pd}$ ( $n=6$ protons) isotones include states of seniority $v=4$ as well as $v=0,2$. In the USp(10) model, the lowest-energy $J$ $=2,4,8$ states fix the energy parameters $(a, b, c)$ of the Hamiltonian (64), cf. Table VI. Eq. (65) then predicts the energies of all other $(9 / 2)^{n}$ states of these nuclei as listed in Tables VII and VIII. The errors for the predicted energies of the $J=6$ levels in the USp(10) approximation are $0.6 \%$ for ${ }^{94} \mathrm{Ru}$ and $2.1 \%$ for ${ }^{96} \mathrm{Pd}$. The predicted energies of the $J$ $=J_{\max }=12$ and $J=10$ states are calculated analytically from Eqs. (72) and (73). The differences between the predicted $\mathrm{USp}(10)$ energy and the experimental energy for the $J=10$ and $J=12$ levels of ${ }^{94} \mathrm{Ru}$ and ${ }^{96} \mathrm{Pd}$ are small-an indication that seniority is a rather good quantum number for the lowenergy states of these isotones. Another region to test seniority is the $N=126$ isotones: ${ }^{210} \mathrm{Po},{ }^{212} \mathrm{Rn}$, and ${ }^{214} \mathrm{Ra}$. These nuclei have an active proton $\mathrm{g}_{9 / 2}$ shell, and Eq. (65) determines the energies of their excited states when the interaction conserves seniority. Table IX compares the predicted USp(10) energy and the experimental energy of the $J=6$ state when the Hamiltonian parameters are fixed by the ex-

TABLE VII. Energy levels in $\mathrm{keV}$ of ${ }^{94} \mathrm{Ru}$ positive parity states. The theoretical levels are calculated in the $\operatorname{USp}(10)$ and $\mathrm{U}(10)$ approximations.

\begin{tabular}{lccccc}
\hline \hline \multicolumn{5}{c}{$\mathrm{USp}(10)$} & Theory \\
$J$ & $v=0$ & $v=2$ & $v=4$ & $\mathrm{U}(10)$ Theory & Experiment \\
\hline 0 & 0 & & 3574 & 0,3600 & 0 \\
2 & & 1431 & 2772 & 1431,2777 & 1431 \\
3 & & & 3546 & 3567 & \\
4 & & 2187 & 2362,3527 & $2187,2354,3546$ & 2187 \\
5 & & & 3220 & 3228 & \\
6 & & 2483 & 2780,3824 & $2498,2775,3841$ & 2498 \\
7 & & & 3281 & 3280 & \\
8 & & 2645 & 3986 & 2645,3997 & 2645 \\
9 & & 4306 & 4318 & \\
10 & & 4073 & 4074 & 3992 \\
12 & & 4948 & 4953 & 4717 \\
\hline \hline
\end{tabular}


TABLE VIII. Energy levels in $\mathrm{keV}$ of ${ }^{96} \mathrm{Pd}$ positive parity states. The theoretical levels are calculated in the $\operatorname{USp}(10)$ and $\mathrm{U}(10)$ approximations.

\begin{tabular}{lccccc}
\hline \hline \multicolumn{5}{c}{$\mathrm{USp}(10)$} & Theory \\
$J$ & $v=0$ & $v=2$ & $v=4$ & $\mathrm{U}(10)$ Theory & Experiment \\
\hline 0 & 0 & & 3354 & 0,3501 & 0 \\
2 & & 1415 & 2636 & 1415,2719 & 1415 \\
3 & & & 3334 & 3464 & \\
4 & & 2099 & 2275,3320 & $2099,2312,3443$ & 2099 \\
5 & & & 3049 & 3137 & \\
6 & & 2373 & 2659,3595 & $2424,2703,3715$ & 2424 \\
7 & & & 3113 & 3178 & \\
8 & & 2531 & 3752 & 2531,3852 & 2531 \\
9 & & 4047 & 4148 & \\
10 & & 3846 & 3911 & 3784 \\
12 & & 4650 & 4728 & 4574 \\
\hline \hline
\end{tabular}

perimental energies of the $J=2,4,8$ levels. The configuration spaces for ${ }^{212} \mathrm{Rn}$ and ${ }^{214} \mathrm{Ra}$ are the direct sums of $v=0,2,4$ irreps of $\operatorname{USp}(10)$. The $J=2,4,6,8$ yrast states of these isotones are interpreted as pure $v=2$ vectors when the interaction conserves seniority. The experimental energies of the $J$ $=2,4,8$ yrast levels determine the Hamiltonian parameters $a, b, c$ of Eq. (65) and, thereby, predict the energies of the $J=6, J=10$, and $J=12$ states. The energies of all the states of the configuration spaces for ${ }^{212} \mathrm{Rn}$ and ${ }^{214} \mathrm{Ra}$ are reported in Tables X and XI. The small differences between the predicted USp(10) levels and the experimental energies indicate that seniority-conserving terms dominate the Hamiltonians for these isotones.

\section{SENIORITY-MIXING INTERACTIONS}

When the potential mixes seniority, $\operatorname{USp}(2 j+1)$ dynamical symmetry is broken and the full $j$-shell model space is active. The configuration space $(j)^{n}$ carries the fully antisymmetric irrep of $\mathrm{SU}(2 j+1)$. For $j=\frac{9}{2}, \frac{11}{2}, \frac{13}{2}$ (see Table IV), there is one independent seniority-mixing two-body rotational scalar interaction that must be added to the seniorityconserving Hamiltonian. The seniority-breaking term may be taken to be $\hat{Y}=\left[\hat{C}_{2} \otimes \hat{C}_{2}\right]_{0}$. To determine the matrix elements of $\hat{Y}$, the matrix elements of the $\mathrm{SU}(2 j+1)$ operator

TABLE IX. Energy levels in $\mathrm{keV}$ of ${ }^{210}$ Po positive parity states. The theoretical levels are calculated in the USp(10) approximation.

\begin{tabular}{lcrc}
\hline \hline \multicolumn{2}{c}{$\mathrm{USp}(10)$ Theory } \\
$J$ & $v=0$ & $v=2$ & Experiment \\
\hline 0 & 0 & & 0 \\
2 & & 1181 & 1181 \\
4 & 1427 & 1427 \\
6 & 1516 & 1473 \\
8 & 1557 & 1557 \\
\hline \hline
\end{tabular}

TABLE X. Energy levels in $\mathrm{keV}$ of ${ }^{212} \mathrm{Rn}$ positive parity states. The theoretical levels are calculated in the $\operatorname{USp}(10)$ and $U(10)$ approximations.

\begin{tabular}{lccccc}
\hline \hline \multicolumn{5}{c}{ USp(10) Theory } \\
$J$ & $v=0$ & $v=2$ & $v=4$ & $\mathrm{U}(10)$ Theory & Experiment \\
\hline 0 & 0 & & 2383 & 0,2432 & 0 \\
2 & & 1274 & 2167 & 1274,2180 & 1274 \\
3 & & & 2390 & 2430 & \\
4 & & 1502 & 2071,2395 & $1502,2059,2430$ & 1502 \\
5 & & & 2322 & 2338 & \\
6 & & 1611 & 2215,2504 & $1640,2207,2539$ & 1640 \\
7 & & & 2372 & 2375 & \\
8 & & 1694 & 2588 & 1694,2610 & 1694 \\
9 & & & 2699 & 2723 & \\
10 & & & 2660 & 2663 & 2655 \\
12 & & & 2960 & 2972 & 2881 \\
\hline \hline
\end{tabular}

$\hat{C}_{2}$ are needed; they are determined by a straightforward generalization of the numerical methods of Sec. IV B.

In Tables VII, VIII, X, and XI, the theoretical energies of ${ }^{94} \mathrm{Ru},{ }^{96} \mathrm{Pd},{ }^{212} \mathrm{Rn}$, and ${ }^{214} \mathrm{Ra}$ are reported in the $\mathrm{U}(10)$ theory. The Hamiltonian parameters are chosen to give an exact fit to experiment for the energies of the $J=2,4,6,8$ yrast states. These parameters yield predictions for the energies of all other states in the configuration spaces. The changes in the energy spectra in $\mathrm{U}(10)$ from the seniorityconserving model are rather small. Moreover, although the energy of the lowest $J=6$ state is brought into agreement with the experimentally observed energy, the energies of the $J=10$ and 12 states are worsened. Thus, little improvement is achieved by extending the dynamical symmetry from $\mathrm{USp}(10)$ to $\mathrm{U}(10)$. Neither the $\mathrm{USp}(10)$ nor $\mathrm{U}(10)$ energies agree exactly with experiment because the model space is limited to a pure $(j)^{n}$ configuration. To improve results the

TABLE XI. Energy levels in $\mathrm{keV}$ of ${ }^{214} \mathrm{Ra}$ positive parity states. The theoretical levels are calculated in the USp(10) and $U(10)$ approximations.

\begin{tabular}{|c|c|c|c|c|c|}
\hline \multirow[b]{2}{*}{$J$} & \multicolumn{3}{|c|}{ USp(10) Theory } & \multirow[b]{2}{*}{ U(10) Theory } & \multirow[b]{2}{*}{ Experimen } \\
\hline & $v=0$ & $v=2$ & $v=4$ & & \\
\hline 0 & 0 & & 2594 & 0,2689 & 0 \\
\hline 2 & & 1382 & 2355 & 1382,2381 & 1382 \\
\hline 3 & & & 2605 & 2681 & \\
\hline 4 & & 1639 & 2251,2612 & $1639,2228,2681$ & 1639 \\
\hline 5 & & & 2533 & 2564 & \\
\hline 6 & & 1767 & 2416,2739 & $1820,2401,2806$ & 1820 \\
\hline 7 & & & 2594 & 2600 & \\
\hline 8 & & 1865 & 2838 & 1865,2882 & 1865 \\
\hline 9 & & & 2966 & 3012 & \\
\hline 10 & & & 2927 & 2933 & 2944 \\
\hline 12 & & & 3272 & 3293 & 3256 \\
\hline
\end{tabular}


model space should be augmented with other $j$ shells plus neutron and proton core-excited basis states.

\section{CONCLUSION}

The partially-solvable USp $(2 j+1)$ model poses an interesting group theoretical problem because, although its irreps have many states that are labeled uniquely by good quantum numbers, analytical expressions are available for only a restricted subset of the corresponding energy eigenvalues. The possibility of deriving the energies $E(n v J)$ of general multiplicity-free states by some, as yet undiscovered, algebraic method is a significant challenge. However, for the $\mathrm{USp}(2 j+1)$ states that are observed experimentally as of this writing, we have reported formulas for their energy eigenvalues.

The numerical technique of this paper to calculate matrix elements of a Lie algebra irrep may be useful for other Lie algebras. For the classical Lie algebras, the matrix elements are well known in bases that are adapted to canonical chains. However, more sophisticated methods are needed when the matrices are required in bases that do not correspond to a canonical chain. Vector coherent state methods [19] provide algorithms for handling many such cases. But, as they stand, they are not capable of handling the irreps of high rank algebras such as the $\mathrm{USp}(2 j+1)$ and $\mathrm{U}(2 j+1)$ algebras we have considered. We plan to apply our numerical method to other Lie algebraic problems in the future.

\section{ACKNOWLEDGMENTS}

The authors are pleased to acknowledge helpful advice and suggestions from I. Talmi and J. Repka. We are particularly indebted to R. E. Howe for bringing Burnside's theorem to our attention.
[1] Y. Alhassid and A. Leviatan, J. Phys. A 25, L1265 (1992); A. Leviatan, Phys. Rev. Lett. 77, 818 (1996).

[2] I. Talmi, Simple Models of Complex Nuclei (Harwood, Chur, 1991).

[3] K. Helmers, Nucl. Phys. 23, 594 (1961).

[4] D.J. Rowe and G. Rosensteel, Phys. Rev. Lett. 87, 172501 (2001).

[5] B.H. Flowers, Proc. R. Soc. London, Ser. A 212, 248 (1952).

[6] R. Howe, Dual Pairs in Physics: Harmonic Oscillators, Photons, Electrons, and Singletons, Lectures in Applied Math. Vol. 21 (American Mathematical Society, Providence, RI, 1985), p. 179.

[7] T. Ton-That, Contemp. Math. 191, 205 (1995).

[8] M. Moshinsky and C. Quesne, J. Math. Phys. 12, 1772 (1971).

[9] G. Racah, Phys. Rev. 62, 438 (1942); 63, 367 (1943); 76, 1352 (1949).

[10] A. de-Shalit and I. Talmi, Nuclear Shell Theory (Academic Press, New York, 1963).

[11] J. N. Ginocchio and W. C. Haxton, in Symmetries in Science VI, edited by B. Gruber and M. Ramek (Plenum, New York, 1993).
[12] A. G. Ushveridze, Quasi-Exactly Solvable Models in Quantum Mechanics (Institute of Physics, Bristol, 1994).

[13] B. G. Wybourne, Classical Groups for Physicists (Wiley, New York, 1974).

[14] A.K. Kerman, Ann. Phys. (N.Y.) 12, 300 (1961); A.K. Kerman, R.D. Lawson, and M.H. MacFarlane, Phys. Rev. 124, 162 (1961); R.D. Lawson and M.H. MacFarlane, Nucl. Phys. 66, 80 (1965).

[15] A. Arima and H. Kawarada, J. Phys. Soc. Jpn. 19, 1768 (1964); A. Arima and M. Ichimura, Prog. Theor. Phys. 36, 296 (1966).

[16] M. Hamermesh, Group Theory and its Application to Physical Problems (Dover, New York, 1962).

[17] M. J. Carvalho (private communication).

[18] S. Lang, Algebra (Addison-Wesley, New York, 1965), p. 444.

[19] D.J. Rowe and J. Repka, J. Math. Phys. 32, 2614 (1991).

[20] According to the Oxford English Dictionary, the first use of the word "redux" in the sense of "brought back, restored" was made in 1662 by Dryden in Astraea Redux, the title of his poem on the happy restoration and return of His Sacred Majesty. 